Sugata Sen Roy (Calcutta)

Sourav Chakraborty (New Delhi)

\title{
PREDICTION PROBLEMS RELATED TO A FIRST-ORDER AUTOREGRESSIVE PROCESS IN THE PRESENCE OF OUTLIERS
}

Abstract. Outliers in a time series often cause problems in fitting a suitable model to the data. Hence predictions based on such models are liable to be erroneous. In this paper we consider a stable first-order autoregressive process and suggest two methods of substituting an outlier by imputed values and then predicting on the basis of it. The asymptotic properties of both the process parameter estimators and the predictors are also studied.

1. Introduction. In modelling time series data one very often comes across outliers or observations which are different from the rest. These can be caused by recording errors, or more often, by unusual events like changes in economic policies, wars, natural calamities, etc. Thus outliers can be both aberrant observations or precursors of things to come. In either case the outliers can often have a considerable effect on related inferences and can even lead to misspecification of the model itself. Hence the question arises as to how they should be dealt with. Donald and Maddala (1993) suggest one of the following three courses: (i) throw the outliers out, (ii) leave them in, but under control and (iii) change the model.

The problem is more complex for time series data because of the correlations that exist among the successive observations. Outliers in time series data, as Fox (1972) pointed out, can be classified as either an Additive Outlier (AO) or an Innovational Outlier (IO). An AO occurs if a particular observation is aberrant but the succeeding observations are normal, whereas IOs occur when the effect of a large innovation is perpetrated through the

2000 Mathematics Subject Classification: Primary 62M10; Secondary 62M20.

Key words and phrases: autoregressive process, outliers, imputation, estimated predictor. 
dynamics of the model. Since a single aberrant observation is either caused by recording errors or, more often, by some unusual events, it may often be appropriate to take recourse to the "throw them out" method for AOs. However, a persistent change in the observations is often the result of a change in the system or mechanism itself and hence the "change the model" method is generally more suited for the IOs.

Robust methods are routinely employed to estimate the parameters in the presence of outliers. Martin and Yohai (1985) give a detailed discussion of such methods in estimating the ARMA parameters in the presence of outliers. An improved recursive robust estimation method is suggested by McDougall (1994). Chang, Tiao and Chen (1988) used the maximum likelihood method and an iterative procedure to estimate the outliers and the parameters of an $\operatorname{ARMA}(p, q)$ process in the presence of both types of outliers. Bruce and Martin (1989) discussed the deletion technique in identifying outliers in an ARIMA model and suggested a strategy for model fitting. Ljung (1993) showed that estimating additive outliers is directly related to the estimation of deleted observations.

However, very little work has been done on predictions based on a time series having outliers. The predictors depend on the model parameters and hence the estimated predictors depend on the corresponding parameter estimators. These estimators are influenced by the presence of outliers which in turn leads to erroneous predictions. However, deletion of the outliers is not a solution since it breaks down the structure of the model or leads to unequally spaced observations which are difficult to model. Such observations thus need to be substituted by imputed values to restore the structure. This, therefore, has an impact on the estimated predictors and consequently on their performances. It is this that we seek to study in this paper.

We consider the prediction problems related to a stable, first order autoregressive model $(\mathrm{AR}(1))$ in the presence of a single $\mathrm{AO}$. We replace the outlier by an imputed value and then make the prediction. Two different imputation techniques are suggested and the consistency of both the estimators of the process parameters and the estimated predictors are shown. The corresponding mean square errors are also derived.

Consider the $\operatorname{AR}(1)$ model

$$
X_{t}=\alpha X_{t-1}+\varepsilon_{t}, \quad t=1, \ldots, n,|\alpha|<1,
$$

where $X_{t}$ is the observation at time $t, t=1, \ldots, n$, and $\varepsilon_{t}$ 's are a sequence of white noises which are continuous with zero mean and variance $\sigma^{2}$. Also assume that

$$
E\left(\varepsilon_{t}^{\delta}\right)<\infty \text { for any } \delta>2,
$$

and 


$$
\inf P\left[\left|\varepsilon_{t}\right| \geq \nu\right]>0 \quad \text { for some } \nu>0
$$

and define

$$
\gamma(0)=\lim _{n \rightarrow \infty} E\left(X_{n}^{2}\right)
$$

Given $n$ observations and under the assumption of zero initial condition, for any integer $s$, the $s$-steps ahead predictor of $X_{n+s}$ is given by the conditional mean $\bar{X}_{n+s}=E\left(X_{n+s} \mid X_{n}, X_{n-1}, \ldots\right)=\alpha^{s} X_{n}$. However, since $\alpha$ is generally unknown, it needs to be estimated and the corresponding predictor obtained.

An estimator of the unknown parameter $\alpha$ is given by

$$
\widehat{\alpha}=\sum_{t=2}^{n} X_{t} X_{t-1} / \sum_{t=2}^{n} X_{t-1}^{2} .
$$

Now under condition (1.2) and following Lai and Wei (1985), we have

$$
\sum_{t=2}^{n} X_{t-1}^{2}=O(n) \quad \text { a.s. }
$$

and

$$
\sum_{t=2}^{n} X_{t-1} \varepsilon_{t}=O\left((n \log n)^{1 / 2}\right) \quad \text { a.s. }
$$

so that using (1.3) yields

$$
\widehat{\alpha}=\alpha+O\left(n^{-1 / 2}(\log n)^{1 / 2}\right),
$$

i.e. $\widehat{\alpha}$ is a consistent estimator of $\alpha$.

Let $\widehat{\gamma}(0)=n^{-1} \sum_{t=2}^{n} X_{t-1}^{2}$. Then since $\varepsilon_{t}$ 's and hence $x_{t}$ 's are continuous, $n \widehat{\gamma}(0)$ is non-zero with probability one for sufficiently large $n$. Thus from Basu and Sen Roy (1986),

$$
\widehat{\gamma}(0)^{-i}=\gamma(0)^{-i}+O\left(n^{-1}\right) \quad \text { for any } i \geq 1 .
$$

Hence, under condition (1.3), which implies uniform integrability of $\widehat{\gamma}(0)^{-1}$, and using (1.9), we obtain

$$
\operatorname{MSE}(\widehat{\alpha})=\sigma^{2} / n \gamma(0)+O\left(n^{-2}\right) .
$$

The estimated predictor of $X_{n+s}$ is given by $\widehat{X}_{n+s}=\widehat{\alpha}^{s} X_{n}$.

Now by Lai and Wei (1985),

$$
\left|X_{n}\right|=o\left(n^{\beta}\right), \quad \beta>1 / \delta,
$$

so that following Basu and Sen Roy (1986),

$$
\left|\widehat{X}_{n+s}-\bar{X}_{n+s}\right|=o\left(n^{\beta-1 / 2}(\log n)^{1 / 2}\right) \quad \text { a.s. }
$$

Also for any $\delta>\max (4,2 s)$ in (1.2),

$$
\operatorname{MSE}\left(\widehat{X}_{n+s}\right)=\sigma^{2} \frac{1-\alpha^{2 s}}{1-\alpha^{2}}+O\left(n^{-1}\right) .
$$


Next suppose the $k$ th observation $(1<k \leq n)$ is an outlier. This would obviously have an inordinate impact on $\widehat{\alpha}$. In order to negate this, the best method would be to delete $X_{k}$. However, this would disrupt the AR structure. Hence to restore the structure it would be necessary to substitute the missing $k$ th observation by some imputed value. The impact of this is what we seek to discuss in this paper.

We consider two different methods of imputing for the deleted observation. The simplest method is to use the average of the remaining $(n-1)$ observations. A second method would be to predict the deleted observation by fitting an AR(1) model to the observations preceding it. The effects of these imputations on the estimation of the model parameters are then observed and compared through their respective mean square errors.

In Sections 2 and 3 we discuss the imputation based on the two methods, while in Section 4 we provide a numerical comparison. Some concluding remarks are made in Section 5.

2. The Average Replacement Method (ARM). In this method we substitute $X_{k}$ by

$$
\bar{X}_{k}=\sum_{t=1, \neq k}^{n} X_{t} /(n-1) .
$$

With $\bar{X}_{k}$ replacing $X_{k}$, the estimator of $\alpha$ would be

$$
\widehat{\alpha}_{\mathrm{ARM}}=\frac{\sum_{t=2, \neq k, k+1}^{n} X_{t} X_{t-1}+\bar{X}_{k} X_{k-1}+X_{k+1} \bar{X}_{k}}{\sum_{t=2, \neq k+1}^{n} X_{t-1}^{2}+\bar{X}_{k}^{2}}
$$

Theorem 2.1, Under condition (1.2),

$$
\widehat{\alpha}_{\mathrm{ARM}}=\alpha+O\left(n^{-1 / 2}(\log n)^{1 / 2}\right) \quad \text { a.s. }
$$

Also under conditions (1.3) and (1.2) with $\delta \geq 4$,

$$
\operatorname{MSE}\left(\widehat{\alpha}_{\mathrm{ARM}}\right)=\sigma^{2} / n \gamma(0)+O\left(n^{-1}\right) .
$$

Proof. From (2.2),

$$
\begin{aligned}
\widehat{\alpha}_{\mathrm{ARM}} & -\alpha \\
= & \frac{\sum_{t=2}^{n} X_{t-1} \varepsilon_{t}-\left(X_{k}-\bar{X}_{k}\right)\left(X_{k-1}+X_{k+1}-\alpha \bar{X}_{k}-\alpha X_{k}\right)}{\sum_{t=2}^{n} X_{t-1}^{2}+\bar{X}_{k}^{2}-X_{k}^{2}} \\
= & (A-C) / B,
\end{aligned}
$$

where

$$
\begin{aligned}
& A=\sum_{t=2}^{n} X_{t-1} \varepsilon_{t}, \quad B=\sum_{t=2}^{n} X_{t-1}^{2}+\bar{X}_{k}^{2}-X_{k}^{2}, \\
& C=\left(X_{k}-\bar{X}_{k}\right)\left(X_{k-1}+X_{k+1}-\alpha \bar{X}_{k}-\alpha X_{k}\right) .
\end{aligned}
$$


Using (1.4), we obtain

$$
E\left(X_{k}^{2}\right)=\gamma(0), \quad E\left(\bar{X}_{k}^{2}\right)=O\left(n^{-1}\right), \quad E\left(n^{-1} \sum_{t=2}^{n} X_{t-1}^{2}\right) \rightarrow \gamma(0),
$$

and hence, under condition (1.2),

$$
n^{-1} B=\gamma(0)+O\left(n^{-1}\right) \quad \text { a.s. }
$$

Again by (1.7) and (1.4) respectively, $A$ is of order $O\left((n \log n)^{1 / 2}\right)$ while $C$ tends to $\alpha \gamma(0)$, so that

$$
n^{-1}(A-C)=O\left(n^{-1 / 2}(\log n)^{1 / 2}\right) \quad \text { a.s. }
$$

Combining (2.6) and (2.7) yields (2.3).

Next observe that under condition (1.9) and finite fourth-order moments,

$$
n^{2} B^{-2}=\left(\widehat{\gamma}(0)+n^{-1} \bar{X}_{k}^{2}-n^{-1} X_{k}^{2}\right)^{-2}=\gamma(0)^{-2}+O\left(n^{-1}\right) .
$$

Also $E\left(n^{-1} A^{2}\right)=\sigma^{2} \gamma(0)$ and under finite fourth-order moments, $E\left(C^{2}\right)=$ $O(1)$ and $E\left(n^{-1} A C\right)=O(1)$ so that

$$
n^{-2} E(A-C)^{2}=\sigma^{2} \gamma(0) / n+O\left(n^{-1}\right) .
$$

Hence under condition (1.3), using (2.8) and (2.9) we obtain (2.4).

Theorem 2.2. Under condition (1.2),

$$
\left|\widehat{X}_{n+s}^{\mathrm{ARM}}-\bar{X}_{n+s}\right|=o\left(n^{\beta-1 / 2}(\log n)^{1 / 2}\right) \quad \text { a.s. }
$$

Also if $\delta \geq 4 s$ in (1.2),

$$
\operatorname{MSE}\left(\widehat{X}_{n+s}^{\mathrm{ARM}}\right)=\sigma^{2} \frac{1-\alpha^{2 s}}{1-\alpha^{2}}+O\left(n^{-1}\right) .
$$

Proof. Writing

$$
\widehat{\alpha}_{\mathrm{ARM}}^{s}-\alpha^{s}=\sum_{j=0}^{s-1}\left(\begin{array}{l}
s \\
j
\end{array}\right)\left(\widehat{\alpha}_{\mathrm{ARM}}-\alpha\right)^{s-j} \alpha^{j},
$$

(2.10) follows by using (2.12), (2.3) and (1.11) in

$$
\widehat{X}_{n+s}^{\mathrm{ARM}}-\bar{X}_{n+s}=\left(\widehat{\alpha}_{\mathrm{ARM}}^{s}-\alpha^{s}\right) X_{n} .
$$

To prove $(2.11)$, since $\varepsilon_{j}, j=n+1, \ldots, n+s$, are independent of $\widehat{\alpha}_{\mathrm{ARM}}$ and $X_{n}$,

$$
\begin{aligned}
\operatorname{MSE}\left(\widehat{X}_{n+s}^{\mathrm{ARM}}\right) & =E\left[\widehat{X}_{n+s}^{\mathrm{ARM}}-X_{n+s}\right]^{2} \\
& =E\left[\widehat{X}_{n+s}^{\mathrm{ARM}}-\bar{X}_{n+s}+\sum_{j=0}^{s-1} \alpha^{j} \varepsilon_{n+s-j}\right]^{2} \\
& =E\left[\left(\widehat{\alpha}_{\mathrm{ARM}}^{s}-\alpha^{s}\right) X_{n}\right]^{2}+\sigma^{2} \frac{1-\alpha^{2 s}}{1-\alpha^{2}} .
\end{aligned}
$$


Using (2.12) and (2.5) gives

$$
\begin{aligned}
E\left[\left(\widehat{\alpha}_{\mathrm{ARM}}^{s}-\alpha^{s}\right) X_{n}\right]^{2} & =E\left[\sum_{j=1}^{s} \sum_{k=1}^{s}\left(\begin{array}{l}
s \\
j
\end{array}\right)\left(\begin{array}{l}
s \\
k
\end{array}\right) B^{-(j+k)}(A-C)^{j+k} \alpha^{2 s-j-k} X_{n}^{2}\right] .
\end{aligned}
$$

Under condition (1.9) and finite moments of order $4 s$, for $j, k=1, \ldots, s$,

$$
\begin{aligned}
n^{j+k} B^{-j+k} & =\left(\widehat{\gamma}(0)+n^{-1} \bar{X}_{k}^{2}-n^{-1} X_{k}^{2}\right)^{-j+k} \\
& =\gamma(0)^{-j+k}+O\left(n^{-1}\right) .
\end{aligned}
$$

Next by Basu and Sen Roy (1986), $E\left(|A|^{j+k}\right)=O\left(n^{(j+k) / 2}\right)$ and because of finite moments of order $4 s, E\left(|C|^{j+k}\right)=O(1)$. Hence using Minkowski's inequality, for $j, k=1, \ldots, s$, we obtain

$$
\begin{aligned}
E|A-C|^{j+k} & \leq\left[\left(E\left(|A|^{j+k}\right)\right)^{-(j+k)}+\left(E\left(|C|^{j+k}\right)\right)^{-(j+k)}\right]^{j+k} \\
& =O\left(n^{(j+k) / 2}\right) .
\end{aligned}
$$

Since $E\left(X_{n}^{2}\right)=\gamma(0)=O(1)$, under condition (1.3) of uniform integrability, using (2.15) and (2.16) in (2.14), we get

$$
E\left[\left(\widehat{\alpha}_{\mathrm{ARM}}^{s}-\alpha^{s}\right) X_{n}\right]^{2}=O\left(n^{-1}\right) .
$$

Inserting (2.17) in (2.13) gives (2.11).

3. The Forecasting Replacement Method (FRM). In this method we substitute $X_{k}$ by the predicted value of $X_{k}$ based on $X_{1}, \ldots, X_{k-1}$, i.e.

$$
\widetilde{X}_{k}=\widehat{\hat{\alpha}} X_{k-1},
$$

where

$$
\widehat{\widehat{\alpha}}=\sum_{t=2}^{k-1} X_{t} X_{t-1} / \sum_{t=2}^{k-1} X_{t-1}^{2}
$$

is the least squares estimator of $\alpha$ in (1.1) based on the first $k-1$ observations.

With $\widetilde{X}_{k}$ replacing $X_{k}$, the estimator of $\alpha$ based on all the observations would be

$$
\widehat{\alpha}_{\mathrm{FRM}}=\frac{\sum_{t=2, \neq k, k+1}^{n} X_{t} X_{t-1}+\widetilde{X}_{k} X_{k-1}+X_{k+1} \widetilde{X}_{k}}{\sum_{t=2, \neq k+1}^{n} X_{t-1}^{2}+\widetilde{X}_{k}^{2}}
$$

TheOREM 3.1, Under condition (1.2) and for $k / n \rightarrow 1$,

$$
\widehat{\alpha}_{\mathrm{FRM}}=\alpha+O\left(n^{-1 / 2}(\log n)^{1 / 2}\right) \quad \text { a.s. }
$$

Also under conditions (1.3) and (1.2) with $\delta \geq 4$,

$$
\operatorname{MSE}\left(\widehat{\alpha}_{\mathrm{FRM}}\right)=\sigma^{2} / n \gamma(0)+O\left(n^{-1}\right) .
$$


Proof. Using (3.3), we obtain

(3.6) $\widehat{\alpha}_{\mathrm{FRM}}-\alpha$

$$
\begin{aligned}
& =\frac{\sum_{t=2}^{n} X_{t-1} \varepsilon_{t}-\left(X_{k}-\widetilde{X}_{k}\right)\left(X_{k-1}+X_{k+1}-\alpha \widetilde{X}_{k}-\alpha X_{k}\right)}{\sum_{t=2}^{n} X_{t-1}^{2}+\widetilde{X}_{k}^{2}-X_{k}^{2}} \\
& =(A-\widetilde{C}) / \widetilde{B},
\end{aligned}
$$

where

$$
\begin{aligned}
\widetilde{B} & =\sum_{t=2}^{n} X_{t-1}^{2}+\widetilde{X}_{k}^{2}-X_{k}^{2}, \\
\widetilde{C} & =\left(X_{k}-\widetilde{X}_{k}\right)\left(X_{k-1}+X_{k+1}-\alpha \widetilde{X}_{k}-\alpha X_{k}\right) .
\end{aligned}
$$

Now, by Basu and Sen Roy (1986), $\widehat{\widehat{\alpha}}^{2}-\alpha^{2}=O\left(k^{-1 / 2}(\log k)^{1 / 2}\right)$ and $X_{k-1}=$ $o\left(k^{\beta}\right)$. Thus under (1.4),

$$
\begin{aligned}
(3.7) \quad n^{-1} \widetilde{B}= & n^{-1} \sum_{t=2}^{n} X_{t-1}^{2}+n^{-1}\left(\widehat{\widehat{\alpha}}^{2}-\alpha^{2}\right) X_{k-1}^{2}+n^{-1}\left(\alpha^{2} X_{k-1}^{2}-X_{k}^{2}\right) \\
= & \gamma(0)+O\left(n^{-1}\right) o\left(k^{2 \beta}\right) \quad \text { a.s., } \\
(3.8) \quad n^{-1} \widetilde{C}= & n^{-1}\left(X_{k}-(\widehat{\widehat{\alpha}}-\alpha) X_{k-1}-\alpha X_{k-1}\right) \\
& \cdot\left(X_{k-1}+X_{k+1}-\alpha\left(\alpha X_{k-1}+X_{k}\right)-\alpha(\widehat{\widehat{\alpha}}-\alpha) X_{k-1}\right) \\
= & O\left(n^{-1}\right) o\left(k^{2 \beta}\right) \quad \text { a.s., } \\
\text { (3.9) } \quad n^{-1} A= & O\left(n^{-1 / 2}(\log n)^{1 / 2}\right) \quad \text { a.s. }
\end{aligned}
$$

Combining (3.7)-(3.9), under the condition $k / n \rightarrow 1$, we obtain (3.4).

Again under finite fourth-order moments and condition (1.9),

$$
\begin{aligned}
n^{2} \widetilde{B}^{-2} & =n^{2}\left[\sum_{t=2}^{n} X_{t-1}^{2}+n^{-1}\left(\widehat{\widehat{\alpha}}^{2}-\alpha^{2}\right) X_{k-1}^{2}+n^{-1}\left(\alpha^{2} X_{k-1}^{2}-X_{k}^{2}\right)\right]^{-2} \\
& =\left[\gamma(0)-O\left(n^{-1}\right) o\left(k^{2 \beta}\right)\right]^{-2} \\
& =\gamma(0)^{-2}+O\left(n^{-1}\right) o\left(k^{2 \beta}\right) \quad \text { a.s. }
\end{aligned}
$$

Also $E\left(n^{-1} A^{2}\right)=\sigma^{2} \gamma(0)$. From Theorem 2.1 of Basu and Sen Roy (1986), $E\left(X_{k}-\widetilde{X}_{k}\right)^{2}=O(1)$, so that under finite fourth-order moments and using the Cauchy-Schwarz inequality, we obtain

$$
E\left(\widetilde{C}^{2}\right) \leq E\left(X_{k}-\widetilde{X}_{k}\right)^{2} E\left(X_{k-1}+X_{k+1}-\alpha \widetilde{X}_{k}-\alpha X_{k}\right)^{2}=O(1) \quad \text { a.s. }
$$

and $n^{-1} E(A \widetilde{C})=O(1)$ a.s., so that

$$
n^{-2}(A-\widetilde{C})^{2}=\sigma^{2} \gamma(0) / n+O\left(n^{-1}\right) .
$$

Using (3.10) and (3.11) gives (3.5).

THEOREM 3.2. Under condition (1.2) and for $k / n \rightarrow 1$,

$$
\left|\widehat{X}_{n+s}^{\mathrm{FRM}}-\bar{X}_{n+s}\right|=o\left(n^{\beta-1 / 2}(\log n)^{1 / 2}\right) \quad \text { a.s. }
$$


Also if $\delta \geq 4 s$ in (1.2),

$$
\operatorname{MSE}\left(\widehat{X}_{n+s}^{\mathrm{FRM}}\right)=\sigma^{2} \frac{1-\alpha^{2 s}}{1-\alpha^{2}}+O\left(n^{-1}\right) .
$$

Proof. The results follow by using arguments similar to those of Theorem 2.2 .

4. A simulation study. Here we illustrate our technique with an example. For this we generated the innovations $\varepsilon_{t}$ as a sample of size $n=100$ from a standard normal distribution. Then the observations, $X_{1}, \ldots, X_{100}$, were obtained by using the model $X_{t}=\alpha X_{t-1}+\varepsilon_{t}$ with $X_{0}=0$ and $\alpha=0.6$. The estimated value of $\alpha$ based on all the 100 observations came out as $\widehat{\alpha}=0.6888$.

Table 4.1. Five-steps ahead predicted values using ARM (upper values) and FRM (lower values) obtained for different $n$

\begin{tabular}{|c|c|c|c|c|c|c|}
\hline$n$ & $\alpha$ & $X_{101}$ & $X_{102}$ & $X_{103}$ & $X_{104}$ & $X_{105}$ \\
\hline full data & 0.6888 & -0.8002 & -0.5512 & -0.3796 & -0.2615 & -0.1801 \\
\hline 10 & 0.6832 & -0.7937 & -0.5423 & -0.3705 & -0.2531 & -0.1729 \\
& 0.4866 & -0.5653 & -0.2751 & -0.1339 & -0.0651 & -0.0317 \\
\hline 20 & 0.6822 & -0.7926 & -0.5407 & -0.3689 & -0.2517 & -0.1717 \\
& 0.7639 & -0.8875 & -0.6779 & -0.5178 & -0.3956 & -0.3022 \\
\hline 30 & 0.6824 & -0.7928 & -0.5410 & -0.3692 & -0.2520 & -0.1719 \\
& 0.6950 & -0.8075 & -0.5612 & -0.3900 & -0.2711 & -0.1884 \\
\hline 40 & 0.6825 & -0.7929 & -0.5411 & -0.3693 & -0.2521 & -0.1720 \\
& 0.6483 & -0.7532 & -0.4883 & -0.3166 & -0.2053 & -0.1331 \\
\hline 50 & 0.6828 & -0.7932 & -0.5416 & -0.3698 & -0.2525 & -0.1724 \\
& 0.6182 & -0.7182 & -0.4440 & -0.2745 & -0.1697 & -0.1049 \\
\hline 60 & 0.6830 & -0.7935 & -0.5420 & -0.3702 & -0.2529 & -0.1727 \\
& 0.6014 & -0.6987 & -0.4202 & -0.2527 & -0.1520 & -0.0914 \\
\hline 70 & 0.6828 & -0.7932 & -0.5416 & -0.3698 & -0.2525 & -0.1724 \\
& 0.6013 & -0.6986 & -0.4200 & -0.2525 & -0.1519 & -0.0913 \\
\hline 80 & 0.6827 & -0.7931 & -0.5414 & -0.3696 & -0.2523 & -0.1722 \\
& 0.6146 & -0.7140 & -0.4388 & -0.2697 & -0.1658 & -0.1019 \\
\hline 90 & 0.6831 & -0.7936 & -0.5421 & -0.3703 & -0.2529 & -0.1728 \\
& 0.7130 & -0.8283 & -0.5906 & -0.4211 & -0.3002 & -0.2140 \\
\hline 100 & 0.6830 & -0.7935 & -0.5420 & -0.3702 & -0.2528 & -0.1727 \\
& 0.6862 & -0.7972 & -0.5470 & -0.3754 & -0.2576 & -0.1768 \\
\hline & & & & & & \\
\hline
\end{tabular}

However, when one of the observations, in this case the 97th, is contaminated by adding or subtracting a constant to make it an outlier, the $\alpha$ value 
changes drastically from 0.6888 . The parameter $\alpha$ is then estimated by deleting the 97th observation and using in turn the estimators $\widehat{\alpha}_{\text {ARM }}$ and $\widehat{\alpha}_{\text {FRM }}$ instead of $\widehat{\alpha}$. Predictions for the next five periods, i.e. for $X_{101}, \ldots, X_{105}$, are then made.

For prediction, the sample size can only be increased by bringing in more past observations. So we start with the last 10 of the 100 observations, i.e. $X_{91}, \ldots, X_{100}$, then the last 20 , i.e. $X_{81}, \ldots, X_{100}$, and so on.

As can be seen from the table, both the estimators and the predictors converge to the original values for large $n$ in both cases, although the convergence under ARM is much faster than under FRM.

5. Concluding remarks. Theorems 2.1 and 3.1 show that, like $\widehat{\alpha}$, the estimators $\widehat{\alpha}_{\mathrm{ARM}}$ and $\widehat{\alpha}_{\mathrm{FRM}}$ converge to $\alpha$. However, the convergence of their mean square errors is much slower than that of $\widehat{\alpha}$. The estimators of the predictors and their mean square errors are the same for ARM and FRM as for the case of all observations. Of course, for the FRM all the above results are true only if $k / n \rightarrow 1$. For $k$ small and fixed, the rates would depend on $k$ and FRM will not be as efficient as ARM.

Since the above methods do not depend on the actual outlying observation, which is deleted and the truncated set of observations used for both estimation and prediction, the methods are equally applicable while fitting an $\mathrm{AR}(1)$ model to a set of observations with a missing value.

This paper can be extended to a general $\operatorname{AR}(p)$ process and further to a moving average process of order $q(\operatorname{MA}(q))$, or even to an $\operatorname{ARMA}(p, q)$ model. However, for the $\operatorname{MA}(q)$ or $\operatorname{ARMA}(p, q)$ models the dependence of the estimators and the predictors on the unknown innovations causes considerable difficulty and the innovations themselves need to be estimated before predictions can be done. For an $\mathrm{MA}(q)$ process, the estimation problems have been studied, among others, by Chan and Tsay (1996) and the prediction problem by Sen Roy (2002). But these problems are liable to be much more complex in the presence of outliers. The complexity would further increase if for an $\operatorname{AR}(p)$ or an $\operatorname{MA}(q)$ the outlier is one of the last $p$ or $q$ observations.

\section{References}

[1] A. K. Basu and S. Sen Roy (1986), On some asymptotic results for multivariate autoregressive models with estimated parameters, Cal. Statist. Assoc. Bull. 35, 139140, 123-132.

[2] A. G. Bruce and R. D. Martin (1989), Leave-k-out diagnostics for time series, J. Roy. Statist. Soc. Ser. B 51, 363-424.

[3] N. H. Chan and R. S. Tsay, (1996), Asymptotic inference for non-invertible moving average time series, J. Time Series Anal. 17, 1-17. 
[4] I. Chang, G. C. Tiao and C. Chen (1988), Estimation of time series parameters in the presence of outliers, Technometrics 30, 193-204.

[5] S. G. Donald and G. S. Maddala (1993), Identifying outliers and influential observations in econometric models, in: Handbook of Statistics, Vol. 11: Econometrics, G. S. Maddala, C. R. Rao and H. D. Vinod (eds.), North-Holland, Amsterdam, 663-701.

[6] A. J. Fox (1972), Outliers in time series, J. Roy. Statist. Soc. Ser. B 34, 350-363.

[7] T. L. Lai and C. Z. Wei (1985), Asymptotic properties of multivariate weighted sums with applications to stochastic regression in linear dynamic systems, in: Multivariate Analysis VI, P. R. Krishnaiah (ed.), North-Holland, Amsterdam, 375-393.

[8] G. M. Ljung (1993), On outlier detection in time series, J. Roy. Statist. Soc. Ser. B $55,559-567$.

[9] R. D. Martin and V. J. Yohai (1985), Robustness in time series and estimating ARMA models, in: Handbook of Statistics, Vol. 5, E. J. Hannan, D. R. Brillinger and P. R. Krishnaiah (eds.), Elsevier, New York, 119-155.

[10] A. J. McDougall (1994), Robust methods for recursive autoregressive moving average estimation, J. Roy. Statist. Soc. Ser. B 56, 189-207.

[11] S. Sen Roy (2002), Predictions in an invertible moving average process with martingale difference innovations, Stochastic Modelling Appl. 5, 46-53.

Department of Statistics

University of Calcutta

35, Ballygunge Circular Road

Calcutta 700019, India

E-mail: ssrstat@caluniv.ac.in
Social Statistics Division Central Statistical Organisation Ministry of Statistics \& Programme Implementation Government of India West Block 8, Wing 6, Sector 1, R.K. Puram

New Delhi 110066, India E-mail: bandhu_kesh@rediffmail.com

Received on 19.10.2006;

revised version on 27.12.2006 